likely to use a condom than those in CNB $(14.1 \%$ vs $5.5 \%$, [age adjusted] $\mathrm{AOR}=0.32$, 95\%CI:0.18-0.56). In URB, condom use was associated with being male (16.7\% vs $12.2 \%$ female, [age/region adjusted] $\mathrm{AOR}=1.47$, 95\%CI:1.12-1.94) and weakly associated with being older (16.5\% among 17-19y vs $12.9 \%$ among $14-16 y$, $\mathrm{AOR}=1.27$, 95\% CI: 0.97-1.68, $\mathrm{p}=0.08)$. In $\mathrm{CNB}$, condom use was associated with being female $(10.5 \%$ vs $1.7 \%$ male, $\mathrm{AOR}=0.16$, 95\% CI: $0.04-$ 0.58). Among URB participants, condom use was associated with sexual activity within the previous month $(21.0 \%$ vs $4.4 \%$ non-recent activity, $\mathrm{AOR}=2.26,95 \% \mathrm{CI}: 1.37-3.74)$. In univariable analysis, participants who reported sexual debut at $\geq 15 \mathrm{y}(17.9 \%)$ were more likely to report condom use than $<15$ y $(17.9 \%, O R=1.24,95 \% \mathrm{CI}: 0.95-1.62, \mathrm{p}=0.08)$.

Conclusions Reported condom use at last sexual encounter was low among adolescents in URB and CNB schools, particularly among adolescents of both sexes in CNB. Younger adolescents, those with a lower age of sexual debut, and especially females in URB and males in $\mathrm{CNB}$, require targeted interventions to increase condom use at all encounters.

\section{P229 PREVALENCE OF MACROLIDE AND TETRACYCLINE RESISTANT TREPONEMA PALLIDUM STRAINS IN SYPHILIS CASES, LIMA AND PUCALLPA, PERU}

${ }^{1} \mathrm{M}$ Eguiluz ${ }^{*},{ }^{1} S$ Vargas, ${ }^{2} \mathrm{~L}$ Giacani, ${ }^{3} \mathrm{M}$ Reyes-Diaz, ${ }^{4} \mathrm{~K}$ Konda, ${ }^{3} \mathrm{C}$ Caceres, ${ }^{4} \mathrm{~J}$ Klausner. ${ }^{1}$ Sexual Health Laboratory, Center for Interdisciplinary Studies in Sexuality, AIDS and Society, Universidad Peruana Cayetano Heredia, Lima, Peru; ${ }^{2}$ Department of Medicine, Division of Allergy and Infectious Diseases, University of Washington, Seattle, USA; ${ }^{3}$ Center for Interdisciplinary Studies in Sexuality, AIDS and Society, Lima, Peru; ${ }^{4}$ Division of Infectious Diseases, David Geffen School of Medicine, University of California, Los Angeles, USA

10.1136/sextrans-2021-sti.314

Background Genetic resistance to macrolides has become widespread in Treponema pallidum subspecies pallidum (TP), while tetracyclines continue to be useful. Alternatives to injectable penicillin are needed globally, given outages and possible allergies. Monitoring of the frequency of resistance in TP isolates remains important for both classes of antibiotics, as unregulated antibiotic use could limit the utility of tetracyclines and macrolide antibiotics like azithromycin in Peru and many countries. Our objective was to determine the frequency of macrolide and tetracycline resistance in TP-positive lesion samples from syphilis cases at Peruvian sexual health clinics.

Methods We analyzed 58 lesion swab samples from patients diagnosed with active syphilis from five STI clinics in Lima and Pucallpa, Peru, from 2019-2020. We extracted DNA from clinical specimens and amplified tp0548 and tp0574 genes to detect the presence of TP. For macrolide resistance, we performed restriction fragment length polymorphism of the 23S rRNA gene to detect the A2058G and A2059G mutations. For tetracycline resistance, we analyzed the G1058C point mutation in the $16 \mathrm{~S}$ rRNA sequences assembled from whole-genome sequencing and compared them with the standard TP Nichols strain sequence using snippy v.2 software.

Results Among the 58 lesion samples analyzed, 25 (44\%) were positive for TP DNA. Among those, we found the A2058G macrolide resistance mutation in 22 samples (88\%); none $(0 \%)$ had the A2059G mutation. None $(0 \%)$ of six $16 \mathrm{~S}$ rRNA sequences analyzed contained the $\mathrm{G} 1058 \mathrm{C}$ point mutation for tetracycline resistance.

Conclusions The frequency of macrolide-resistance mutations in these TP isolates remains elevated, confirming that macrolides should not be used to treat syphilis. Conversely, as resistance to tetracyclines was absent, corroborating that tetracyclines continue to be useful as a safe and effective alternative for syphilis treatment in this context.

\section{P232 ANTIMICROBIAL RESISTANCE OF NEISSERIA GONORRHOEAE IN GERMANY 2016-2020, RESULTS FROM THE GONOCOCCAL RESISTANCE NETWORK (GORENET)}

${ }^{1} \mathrm{R}$ Selb*, ${ }^{1} \mathrm{~K}$ Jansen, ${ }^{2} \mathrm{P}$ Kreher, ${ }^{1} \mathrm{G}$ Zuelsdorf, ${ }^{3} \mathrm{E}$ Guhl, ${ }^{3} \mathrm{I}$ Graeber, ${ }^{1} \mathrm{~V}$ Bremer, ${ }^{2} \mathrm{~S}$ Banhart, ${ }^{2} \mathrm{D}$ Heuer, ${ }^{2,3}{ }^{2}$ Buder. ${ }^{1}$ Department for Infectious Disease Epidemiology, Robert Koch Institute, Berlin, Germany; ${ }^{2}$ Department of Infectious Diseases, Robert Koch Institute, Berlin, Germany; ${ }^{3}$ German Reference Laboratory for Gonococci, Department of Infectious Diseases, Robert Koch Institute, Berlin, Germany

\subsection{6/sextrans-2021-sti.315}

Background In Germany, Neisseria gonorrhoeae (NG) infections are not reportable and limited data on epidemiology and antimicrobial resistance (AMR) are available. Within the Gonococcal Resistance Network (GORENET) we monitor the NGAMR in Germany to inform treatment guidelines.

Methods Between 2016-2020, 75 laboratories sent NG-isolates to the German reference laboratory which were tested for ceftriaxone, cefixime, azithromycin, ciprofloxacin, and penicillin susceptibility using MIC-strip test. AMR-results were interpreted according to the EUCAST breakpoints version 11.0. The proportion of resistant isolates was calculated.

Results As of December 2020, 2437 isolates were tested, $89.2 \%$ were from men. Median age was 33 (men) and 29 years (women). Most isolates were urethral (77.7\%), followed by cervical $(4.6 \%)$ and rectal samples $(4.5 \%)$.

One sample in 2018 was resistant to ceftriaxone (2018: $0.2 \%)$. Resistance to azithromycin was increasing from 20162020 (2016: 0.8\%, 2017:1.0\%, 2018: 3.7\%, 2019: 6.6\%, 2020: 12.8\%). Cefixime resistance was stable (2016: $0.6 \%$; 2017: 0.6\%, 2018: 0.9\%; 2019: 1.9\%, 2020: 0.8\%).

On average, $60.4 \%$ of samples were ciprofloxacin- (2016: 54.3\%; 2017: 63.8\%, 2018: 58.5\%; 2019: 66.2\%, 2020: $59.5 \%)$ and $17.4 \%$ penicillin-resistant (2016: 16.1\%; 2017: 15.1\%, 2018: 17.0\%; 2019: 21.2\%, 2020: 17.9\%).

Conclusion Comparable to other Western countries, the majority of isolates originated from men. The first-line substance ceftriaxone remains effective in Germany, whereas resistance to the important second first-line therapy component azithromycin reached high levels, nearly doubling from 2019 to 2020. Thus, ceftriaxone monotherapy is also recommended in Germany since 2019 if patient adherence is warranted.

Cefixime-resistance remained low considering that the substance is currently not available in Germany. Penicillin and ciprofloxacin resistance was stably high. NG susceptibility testing is crucial to monitor the AMR-situation in Germany and to inform treatment guidelines.

\section{P234 'THERE IS NO MAGIC BULLET.' CONSIDERATIONS FOR ADOPTION OF MOLECULAR CHLAMYDIA AND GONORRHOEA POINT OF CARE TESTS INTO ROUTINE CARE}

${ }^{1} S$ Fuller*, ${ }^{2} E$ Clarke, ${ }^{2} E$ Harding-Esch. 'St George's University of London, London, UK; ${ }^{2}$ London School of Hygiene andTropical Medicine, London, UK

10.1136/sextrans-2021-sti.316 
Background Sexually Transmitted Infections (STIs), including Neisseria gonorrhoeae (NG) and Chlamydia trachomatis (CT), continue to be a global health problem, with the majority of disease burden in Low-and-Middle-Income Countries. This could in part be addressed through increased access to point-of-care-tests (POCTs) to detect infection and appropriately manage cases and contacts. Criteria for the development of STI POCTs have been established, and several CT and NG POCTs have been brought to market. Yet even those diagnostics with good evidence of clinical effectiveness often fail to be implemented and adopted into routine care.

Methods We first reviewed whether the Cepheid CT/NG GeneXpert POCT fulfils published international guidance for STI POCT development: the (RE)ASSURED and Target Product Profile (TPP) criteria. Then, through a systematic review of Medline and Embase of published literature that reported on the test's implementation, demonstrated its values in different settings and to a variety of stakeholders. This information was then applied to form the basis of a value proposition for an 'ideal' CT/NG POCT.

Results The Cepheid CT/NG GeneXpert did not fulfill all (RE)ASSURED or TPP criteria, however, studies of test implementation showed multiple stakeholder values for use of the test across various healthcare settings and locations. The majority of values identified were setting-specific. Sexual health services and outreach services had the least overlap in values, whereas General Practice and other non-sexual health specialist services served as a 'bridge' between the two.

Conclusion We recommend that those wishing to improve CT/ NG diagnosis be supported to identify the values most relevant to their settings and context, and prioritise implementation of those tests most closely aligned with those values.

\section{P235 MAINTAINING ACCESS TO HIV PREP IN A PANDEMIC: PREP-USER AND HEALTH CARE PROFESSIONAL PERSPECTIVES ON A TELEPHONE-BASED PREP SERVICE}

'L Henderson*, 2,3 J Gibbs, 'S Ramasami, 'J Quinn, ${ }^{1,4} \mathrm{C}$ Estcourt. ${ }^{1} \mathrm{NHS}$ Greater Glasgow and Clyde, Glasgow, UK; ${ }^{2}$ Central and North West London NHS Trust, London, UK; ${ }^{3}$ University College London, London, UK; ${ }^{4}$ Glasgow Caledonian University, Glasgow, UK

\subsection{6/sextrans-2021-sti.317}

Background To maintain access to PrEP during the COVID-19 pandemic our PrEP service (1000 PrEP-users) shifted to a largely telephone-based model (tele-PrEP).

Objectives To conduct a service evaluation of tele-PrEP, exploring the views and experiences of PrEP-users and sexual health care professionals (HCPs), to understand benefits and drawbacks to inform future service delivery.

Methods Parallel, web-based, anonymous surveys of PrEP-users and HCPs were developed using validated questions wherever possible. The PrEP-user survey was offered to people who had a tele-PrEP appointment between 13.11.2020-17.12.2020 and consented to participate. All HCPs conducting tele-PrEP appointments were invited to participate. Basic demographic data was captured. Data were analysed in Excel using descriptive statistics. Free text responses were thematically categorised using the Framework for a Systems Approach to Healthcare Delivery.

Results Sixty-two PrEP-users and 8 HCPs completed the surveys (response rate $55 \%$ and $89 \%$ respectively). Demographic characteristics of PrEP-user respondents were broadly representative of our whole PrEP-cohort. Tele-PrEP was rated 'excellent' or 'good' by 61/62 PrEP-users, and 59/62 would recommend it to friends. PrEP-users identified convenience as a key benefit along with access to PrEP with reduced potential for COVID-19 exposure. Drawbacks were largely technological, including poor connection or issues with online booking. All HCPs felt that tele-PrEP allowed them to assess patients safely and confidently. HCPs also rated its convenience highly and felt it enabled better use of limited face-to-face clinic capacity. However, HCPs thought that tele-PrEP might create barriers for vulnerable patients, particularly those with low digital, health and/or English-language literacy. One HCP and 10/62 PrEP-users expressed a personal preference for face-toface appointments.

Conclusion Tele-PrEP is feasible and acceptable. While most respondents rated the service highly, others identified a need/ preference for face-to-face appointments. Therefore, our service will continue tele-PrEP whilst ensuring availability of faceto-face care for those who require or request it.

\section{P236 EXAMINING THE IMPACT OF THE UK'S COVID-19 PUBLIC HEALTH RESPONSE ON SEXUAL BEHAVIOUR AND HEALTH SERVICE USE AMONG MSM}

${ }^{1} \mathrm{~A}$ Howarth, ${ }^{1,2} \mathrm{~J}$ Saunders, ${ }^{1,3} \mathrm{D}$ Reid*, ${ }^{1} \mathrm{I}$ Kelly, ${ }^{1} \mathrm{~S}$ Wayal, ${ }^{3} \mathrm{P}$ Weatherburn, ${ }^{2} \mathrm{G}$ Hughes, ${ }^{1} \mathrm{C}$ Mercer. ' UCL, London, UK; ${ }^{2}$ Public Health England, London, UK; ${ }^{3}$ London School of Hygiene and Tropical Medicine, London, UK

\subsection{6/sextrans-2021-sti.318}

Background The introduction of social distancing in response to the COVID-19 pandemic led to reduced STI/HIV service provision in the UK. We investigated sexual risk behaviours among MSM and unmet need for sexual healthcare during the pandemic.

Methods A cross-sectional online survey $(\mathrm{N}=2,018)$ fielded via social media and dating apps (23/06-14/07/2020). We examined sexual behaviour and service use since lockdown (23/03/ 2020) and in the three previous months, and 'unmet need for STI testing' since lockdown (any new male partners and/or multiple condomless anal sex (CAS) partners without testing for STIs).

We compared behaviours over the past three months between socio-demographically equivalent sub-samples recruited via Grindr into the present survey $(\mathrm{N}=956)$ and a 2017 survey $(\mathrm{N}=1,918)$.

Results In 2020, 36.7\% of participants reported new male partners and $17.3 \%$ reported multiple CAS partners since lockdown. Comparing time since lockdown vs previous three months, HIV testers were less likely to test at sexual health clinics $(22.3 \%$ vs $70.2 \%)$ and more likely to use free online self-sampling services (64.3\% vs $17.1 \%)$, and PrEP users were less likely to report PrEP use (21.7\% vs $65.7 \%)$.

Since lockdown, $25.3 \%$ of participants had unmet need for STI testing. Unmet need was more likely among Asian vs White participants $(\mathrm{aOR}=1.76,[1.14-2.72], \mathrm{p}=.01)$; living in Scotland $(\mathrm{aOR}=2.02,[1.40-2.91], \mathrm{p}<.001)$ or Northern Ireland $(\mathrm{aOR}=1.93,[1.02-3.63], \mathrm{p}=.04)$ vs England; and living with HIV $(\mathrm{aOR}=1.83,[1.32-2.53], \mathrm{p}<.001)$.

Compared to 2017, the 2020 sub-sample were less likely to report new male partners $(46.8 \%$ vs $71.1 \%, \mathrm{p}<.001)$, multiple CAS partners $(20.3 \%$ vs $30.8 \%, \mathrm{p}<.001)$ and unmet need $(32.8 \%$ vs $42.5 \%, \mathrm{p}<.001)$ in the past three months. 